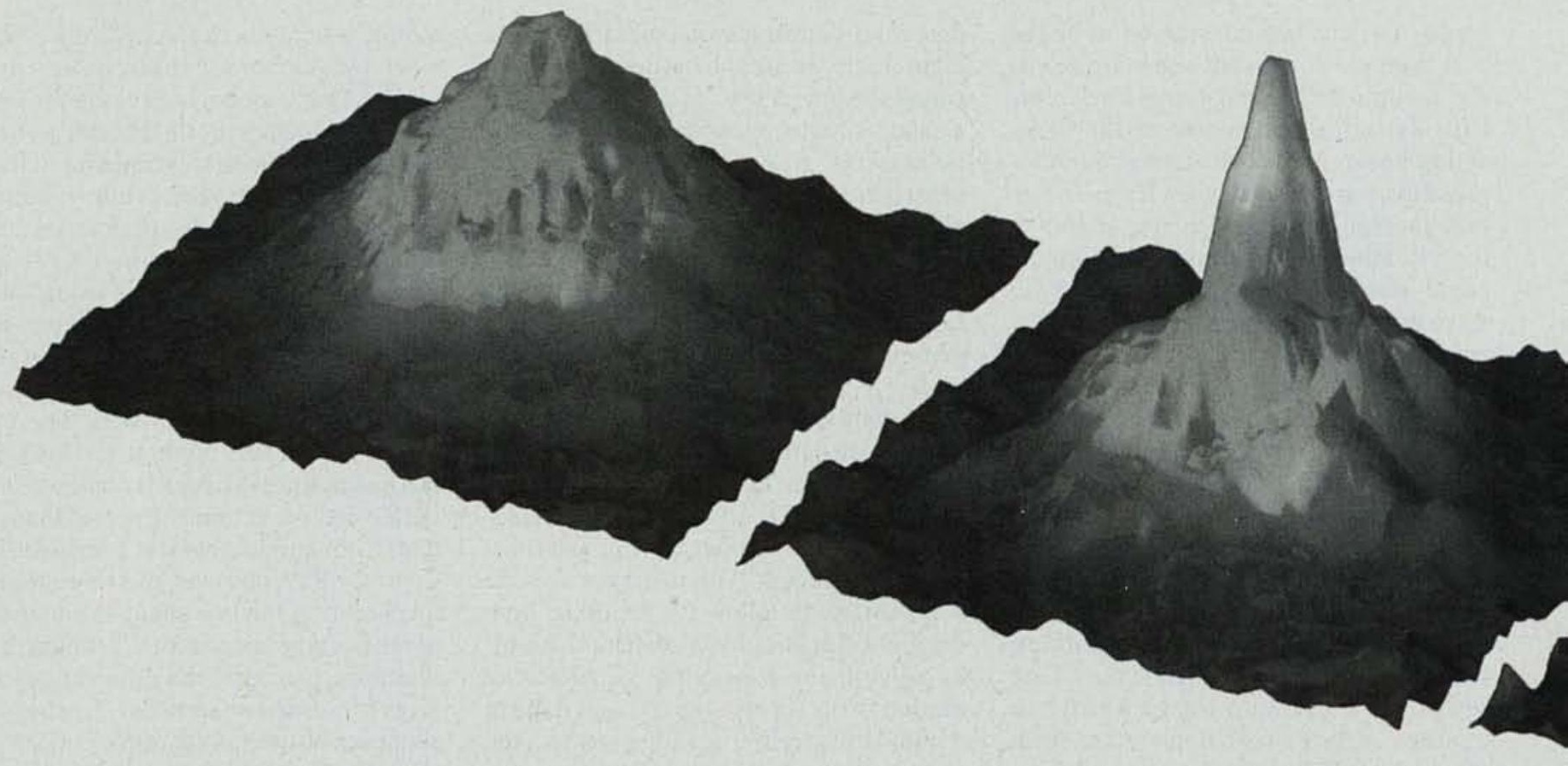

Wojciech Gawlik, Poland

\title{
Bose-Einstein Condensates in Europe
}

Before spring 1995 not many enthusiasts, even among today's experts, believed in obtaining the Bose-Einstein condensation (BEC) of cold atoms in this century, if at all. This bizarre quantum state which is formed at nanokelvin temperatures had been predicted in the twenties. Before 1995 many spectacular successes and record low temperatures had been achieved along the way towards absolute zero-three main pioneers in this field (Steven Chu, Claude Cohen-Tannoudji and Bill Phillips) were honoured with the 1997 Nobel Prize for some of these-but obtaining the $\mathrm{BEC}$ required not only very low temperatures but also high atomic densities and remained a rather elusive objective for many people.

The BEC is formed when the quantum wave packets of particles overlap at low temperatures and the particles condense, almost motionless, into the lowest quantum state. In other words, the wavelengths of the matter waves associated with the particles, the de Broglie waves, become similar in size to the mean particle distances in a cold and dense sample. The first success in achieving this was unexpected. So it was remarkable that the BEC was obtained in three different US laboratories within a few months of summer 1995. First was the group of Eric
Cornell and Carl Wieman (JILA, Boulder, Colorado) working with rubidium atoms, then Randall Hulet and coworkers (Rice University, Houston, Texas) working with lithium and Wolfgang Ketterle (MIT, Cambridge, Massachusetts) who's group worked with sodium. The fact that each of the teams worked with bosonic atoms of different elements indicates adequately that there is a universal character to the phenomenon.

After the above three pioneering experiments an avalanche of further similar experiments was to be expected. This was not to be the case, however. The experimental technique, involving magneto-optical traps, dark-state trapping, evaporation cooling and magnetic traps, was apparently not simple.

The first 'European' Bose-Einstein condensate was obtained in the laboratory of Gerhard Rempe at the University of Konstanz in Germany. Europe had caught up with the US, where in the meantime, in addition to the three first labs, the BEC had been achieved in several other labs: the University of Texas (Austin), the Rowland Institute (Cambridge) and Stanford University in California.

It wasn't very long before other European groups managed to obtain BEC. It was achieved in Munich (Ted Haensch's group) and at Ecole Nationale SupËrieure by Jean Dalibard and coworkers after their switching from caesium to rubidium. Other groups have also now been able to make their rubidium atoms condense: in Hanover (Wolfgang Ertmer's group) and in Orsay (Allan Aspect's team) and most recently at the University of Sussex in Brighton (Malcolm Boshier's and Ed Hinds's group). Though each of the condensates has been obtained using rubidium atoms, there are significant differences between each of the experiments. For example, the Kon$\operatorname{stanz}$ trap allows independent control over the radial and axial confinement, not possible with any other BEC trap, which opens the way to very interesting possibilities, magnetic wave-guiding for example; the Munich team explores magnetic traps of a rather novel shape and the Orsay group uses iron-core magnets in their trap.

Altogether, six European groups have obtained BEC. This elite club is certainly not closed. Many other groups are working very actively on BEC and perhaps by the time you read this further groups will have seen their atoms condense. Many groups are working with cold atoms, laser cooling and trapping in the hope of this.

By now BEC in atomic gases has also 


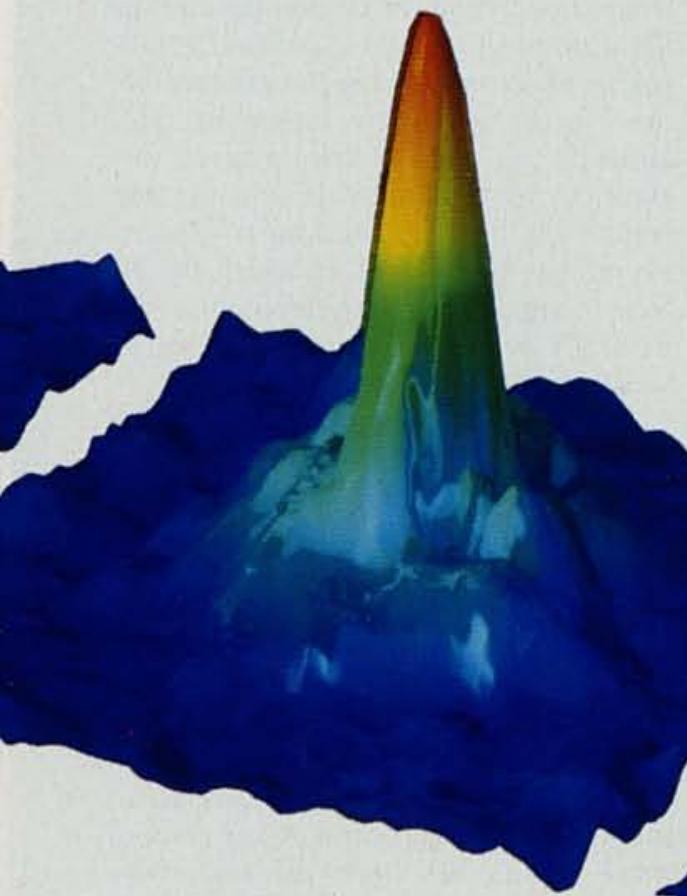

The forming condensate: the density distribution of atoms after free expansion. As the temperature drops from $\mathbf{1 . 2}$ uK left to below 100 uK right, a Bose-Einstein condensate forms at the centre of a gas cloud

SOURCE: EANST, MARTE, SCHRECK, SCHUSter AND REMPE EUROPHYSICS LETTERS 411 (1998)

been obtained in New Zealand (University of Otago), in Japan (University if Tokyo at Komaba and Kyoto University), Gaithersburg (NIST), Yale University, and at MIT by Greytak and Kleppner's group, where the systematic hunt for BEC in atomic hydrogen was initiated a long time ago.

The question of the experimental reality of BEC of free atomic gas is being clearly and positively answered. The BEC has now become a mature and fascinating field of research. The meaning and the potential of this achievement are definitely not limited only to the confirmation of the theoretical prediction of Satyendra Nath Bose and Albert Einstein of 70 years ago. More importantly, as the $\mathrm{BEC}$ is a 'macroscopic' quantum waveform it which makes the usually elusive quantum waveform behaviour visible, and allows us to manipulate it, and so is providing us with samples and techniques for entirely new studies.

This is a very exciting time that could be compared perhaps with the early days of quantum mechanics when atomic physics experiments stimulated profound developments in physics. The properties of the $\mathrm{BE}$ condensate are largely unexplored. Since the condensate has dramatically different properties from independent, uncondensed atoms, every experi- ment carried out on BEC offers new surprises and imposes new questions.

The $\mathrm{BE}$ condensation was seen before in other situations, the superfluidity of helium being the most well known example. Superfluid helium, however, is an ensemble of very dense, strongly interacting atoms. The condensed fraction does not exceed 10 per cent. On the other hand, in the BEC experiments with atomic gases the individual atoms interact very weakly, and the condensed fraction can be very close to 100 per cent.

This offers a unique chance to explore macroscopic properties of these condensates and understand how they are related to microscopic interactions that, in contrast to liquid helium, can be relatively easily controlled and theoretically interpreted. Another exciting subject is to explore the analogy between coherent photons (laser beam) and coherent atoms (BEC). Exploring the analogies between BEC and quantum fluids on the one hand, and laser light on the other hand, will create exciting new fields of research reaching beyond what are now labelled as atomic and laser physics, quantum optics, solid state and statistical physics.

Though the field is very new, many 